\title{
LAS MIGRACIONES INTERNACIONALES Y LA REORGANIZACIÓN DE LOS CUIDADOS. LOS CASOS DE LA REPÚBLICA DE MOLDAVIA, RUMANÍA Y UCRANIA
}

\section{INTERNATIONAL MIGRATIONS AND THE REORGANIZATION OF CARE. THE CASES OF THE REPUBLIC OF MOLDOVA, ROMANIA AND UKRAINE}

Francesca Alice Vianello*

Resumen: En el contexto de los importantes flujos migratorios desde Europa del Este hacia Europa Meridional, en este artículo se presenta un estudio cualitativo realizado en Rumanía, la República de Moldavia y Ucrania en torno a la reorganización de los cuidados que se requieren para afrontar las repercusiones de la ausencia de personas cuidadoras, también conocida como «drenaje de cuidados». En particular, se analiza la reorganización de los cuidados destinados a los menores y a las personas mayores que se quedaron en los países de procedencia tomando en cuenta los cuatro pilares del sistema o polos del «diamante» de los cuidados, a saber: las familias transnacionales, el Estado, el tercer sector y el mercado. La perspectiva desde los países de origen, la comparación entre el sistema de cuidados infantil y el dirigido a los mayores, así como la inclusión del Estado y de la sociedad civil como proveedores de cuidados complementarios a los de las familias, ofrecen una visión más completa de las trasformaciones sociales generadas por la emigración masiva.

Universidad de Padua (Italia), Departamento FISPPA, francescaalice.vianello@unipd.it. 
Palabras clave: migraciones, Europa del Este, drenaje de cuidados, niños, personas mayores.

Abstract: Since the relevance of migrations from Eastern Europe to Southern Europe, this paper presents a qualitative study carried out in Moldova, Romania and Ukraine concerning the reorganization of care to address the impact of care drain. In particular the reorganization of care for children and elderly left behind is analised, taking into account the four poles of the "care diamond»: transnational families, the State, the third sector and the market. The perspective from the countries of origin, the comparison between two systems of care, for childern and for elderly people, and the inclusion of State and civil society into the analysis of care provisions provide a more complete picture of the social transformations generated by mass emigration.

Keywords: migrations, Eastern Europe, care drain, children, older people.

\section{INTRODUCCIÓN}

Uno de los efectos menos visibles de las migraciones internacionales es el fenómeno del «drenaje de cuidados» y en particular sus repercusiones en la sociedad de origen (Ehrenreich, Hochschild 2003; Lutz, Palenga-Möllenbeck 2012). Especialmente cuando son las mujeres quienes emigran y a su vez quienes llevan a cabo gran parte del trabajo de cuidados, sea de forma gratuita o retribuida, se manifiesta en las sociedades de partida lo que algunas estudiosas han definido como déficit o crisis del cuidado, esto es, un empeoramiento de las dificultades para poder garantizar la reproducción social (Laslett \& Brenner, 1989), que se traduce en la ausencia de cuidados para las personas más débiles de la sociedad, como los niños, los ancianos y los discapacitados (Orozco 2010).

En los países de Europa del Este a la situación de déficit de cuidados causada por la emigración de las mujeres se suma el gran retroceso que el sistema de bienestar ha sufrido tras la caída de los sistemas comunistas (Fodor et al., 2002; Tache \& Neesham, 2011; Pascall \& Manning, 2000). Por consiguiente, las actividades necesarias para reproducir la vida vienen a recaer, más que en el pasado, sobre las mujeres de la familia. De todos modos, la emigración de 
las mujeres produce efectos contradictorios, puesto que si bien, por un lado, ellas dejan un vacío difícil de cubrir, por otro lado, con sus remesas garantizan el acceso a la educación superior de sus hijos e hijas, así como la atención médica de las personas mayores.

En el contexto de los importantes flujos migratorios femeninos desde Europa del Este hacia Europa Meridional, en este artículo se presenta un estudio cualitativo realizado en Rumanía, la República de Moldavia y Ucrania en torno a la reorganización de los cuidados que se requiere para afrontar las repercusiones del drenaje de cuidados. En primer lugar, se introduce el marco teórico y la metodología adoptada para la realización de este trabajo. En segundo lugar, se ilustra el fenómeno social de los niños y los ancianos que se quedan sin el sostén físico de sus madres y sus hijas a causa de la emigración. En tercer lugar, se analiza la reorganización social de los cuidados en relación con los menores y las personas mayores que se quedaron en los países de procedencia tomando en cuenta los cuatro pilares del sistema o polos del «diamante» de los cuidados, a saber: las familias transnacionales, el Estado, el tercer sector y el mercado. Puesto que mucho se ha escrito ya acerca de las familias transnacionales, el artículo se centra en dos aspectos específicos que evidencian cómo se reorganizan los cuidados de los niños y de los ancianos: la adaptación del modelo de la familia matrioshka a las nuevas exigencias y las prácticas de cuidado a distancia.

\section{EL MARCO TEÓRICO}

Este artículo presenta los resultados de una investigación cualitativa realizada entre 2011 y 2013 en tres países de Europa del Este —la República de Moldavia, Rumanía y Ucrania- caracterizados por procesos migratorios, en ciertos aspectos, muy similares y profundamente imbricados con los regímenes de cuidado de los países tanto de origen como de destino. En efecto, en los tres casos se trata de migraciones iniciadas tras la caída de las repúblicas socialistas, con una alta participación femenina, dirigidas, en gran medida, hacia países de Europa del Sur como Italia y España ${ }^{1}$, pertenecientes

1 En España se registran 18 mil ciudadanos de nacionalidad moldava (porcentaje de mujeres: 50\%), 89 mil ciudadanos de nacionalidad ucraniana 
al llamado modelo migratorio mediterráneo y atraídas por la elevada demanda de fuerza laboral a emplear en el sector del servicio doméstico y de los cuidados (Bettio et al., 2006; Arango et al., 2009).

El enfoque teórico de la investigación se ha basado en el concepto de transnacionalismo y en la literatura relativa a las cadenas globales del cuidado y a la circulación de los cuidados en las familias transnacionales. Numerosos estudiosos han demostrado que emigrar no comporta necesariamente una ruptura con el contexto socio-cultural de origen, sino que más bien los hombres y las mujeres emigrantes suelen mantener fuertes e intensos lazos con el país de nacimiento y, en particular, con los familiares que se quedaron atrás (Basch et al. 1994; Guarnizo, Smith, 1998; Faist 2000). De este modo, las familias transnacionales son aquellas en las cuales sus miembros a pesar de encontrarse en diferentes países mantienen estrechas relaciones entre sí a lo largo del tiempo y del espacio. El cuidado es uno de los bienes que circula con mayor intensidad dentro de las familias transnacionales y las mujeres emigrantes y no-emigrantes de diferentes generaciones son las principales protagonistas de estos intercambios (Carling et al. 2012; Baldassar et al. 2007; Baldassar, Merla 2013).

La literatura relativa a las migraciones femeninas ha evidenciado la elevada interdependencia entre los regímenes de cuidado de las sociedades de origen y de destino, mostrando cómo las tareas de cuidado realizadas por las personas que emigran para satisfacer la demanda de cuidados de los países del «norte global», se transfieren a quienes permanecen en el «sur global», como las abuelas, las hijas mayores, las tías y, más raramente, los maridos (Williams 2010). Sin embargo si, como sugieren Raghuram (2012) y Herrero (2012), miramos estos fenómenos colocándonos en los países de emigración vemos que los procesos migratorios producen una modificación de la organización social de los cuidados, que involucra no sólo los hogares sino también el Estado, el mercado y el tercer sector.

(porcentaje de mujeres: 56\%) y 843 mil ciudadanos de nacionalidad rumana (porcentaje de mujeres: 48\%) (INE 2012). En cambio en Italia se registran 147 mil ciudadanos moldavos (porcentaje de mujeres: 67\%), 218 mil ciudadanos ucranianos (porcentaje de mujeres: 81\%) y 933 mil ciudadanos rumanos (porcentaje de mujeres: 57\%) (Istat 2011). 
El argumento del texto es que para analizar las transformaciones de las dinámicas de cuidado generadas por la emigración en los países de procedencia no es suficiente con estudiar a las familias migrantes y a la reorganización de los cuidados a nivel transnacional, sino que es necesario incluir en el estudio también las otras instituciones que participan en la gestión de los cuidados. En consecuencia, en este artículo se analizan tanto las prácticas de cuidado de las familias migrantes como la actividad de otras instituciones encargadas de paliar la situación de los miembros de las familias migrantes que se quedaron en Moldavia, Rumanía y Ucrania. En particular, además de los menores, se incluye en el análisis las personas mayores, porque existen diferentes tipos de migraciones femeninas desde Europa del Este: las protagonizadas por madres jóvenes que dejan atrás hijas e hijos menores y las protagonizadas por mujeres con hijas e hijos adultos y padres mayores (Marchetti, Venturini 2013). Por lo tanto, el drenaje de los cuidados no afecta solamente a la categoría de los menores, sino también a las personas mayores.

En este trabajo se adopta la metáfora del «diamante de los cuidados» (Evers 1996; Jenson 2003) para analizar cómo las familias migrantes, el Estado, los mercados locales de cuidados y el tercer sector se están organizando para afrontar las necesidades de los menores y de las personas mayores que viven lejos de sus familiares. Esta metáfora ya fue utilizada por Kofman y Raghuram (2009), para explicar la configuración de los cuidados entre diferentes actores situados tanto en los países de origen como en los de inmigración. Cada uno de los vértices del rombo corresponde a una institución: en primer lugar, las familias y los hogares en todos los países del mundo proporcionan la mayoría de los cuidados; el mercado en cambio ofrece diferentes servicios de cuidados según el contexto social y económico; las comunidades, incluido el tercer sector, tienen un papel cada vez más importante en los servicios de cuidados; por último el Estado (nacional, regional y local) adquiere un papel central en la organización general de los cuidados a través de sus servicios sociales, sanitarios, escolares y las transferencias económicas. 


\section{GRÁFICO 1}

\section{EL DIAMANTE DE LOS CUIDADOS}

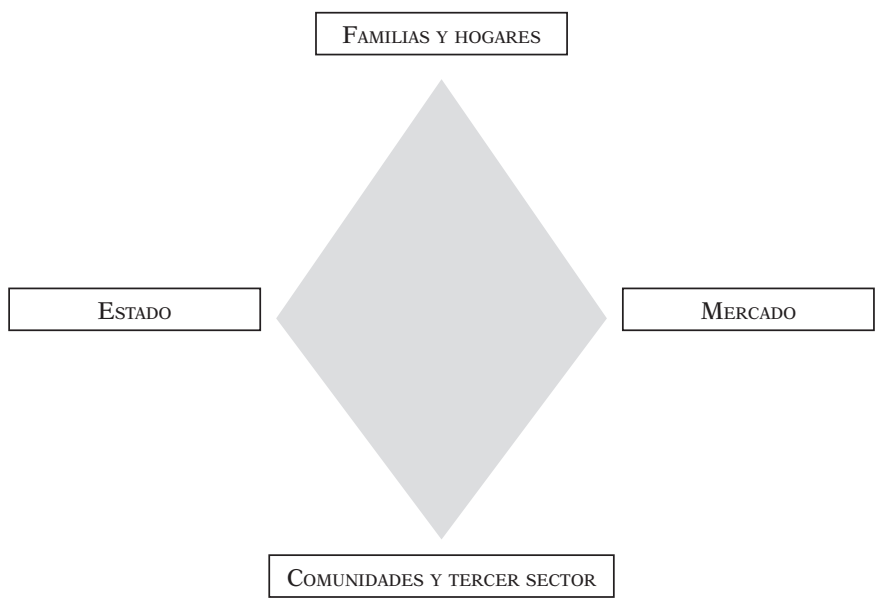

Fuente: Razavi (2007), Kofman, Raghuram, (2009).

Este artículo se basa en datos recogidos entre 2011 y 2013 como parte de una extensa investigación, cualitativa y cuantitativa, sobre la vida cotidiana de las familias transnacionales moldavas, rumanas y ucranianas, prestando especial atención a la reorganización social de los cuidados en los dos extremos del proceso migratorio ${ }^{2}$. En particular, se hace referencia a los datos recogidos durante tres viajes etnográficos realizados entre abril y septiembre de 2012, hasta los distritos de: Chișinău y Drochia (República de Moldavia); Bucarest, Galați y Iași (Rumanía) y L'viv ( Ucrania); en este periodo se realizaron 26 entrevistas a informantes privilegiados ocupados en los servicios escolares, sociales y sanitarios, públicos, privados y del tercer sector (ONG), y 26 entrevistas a familiares de migrantes (Tabla 1).

2 La investigación ha sido financiada por la Universidad de Padua y la Cámara de Comercio de Padua. 
TABLA 1

\begin{tabular}{|l|l|l|}
\hline & Informantes privilegiados & Familiares de migrantes \\
\hline República de Moldavia & 13 & 8 \\
\hline Rumanía & 7 & 9 \\
\hline Ucrania & 6 & 9 \\
\hline Total & 26 & 26 \\
\hline
\end{tabular}

El objetivo del estudio no era evaluar el impacto positivo o negativo de la emigración sobre la sociedad de origen, sino analizar cómo la organización social de los cuidados está cambiando como resultado de la migración internacional. Por tanto, el objetivo de las entrevistas era ver cómo los distintos actores encargados de proporcionar cuidados dentro de un determinado territorio responden a las nuevas necesidades sociales generadas por la emigración masiva.

Durante las entrevistas se discutió no sólo sobre la situación de los hijos de los inmigrantes (una categoría social que ha atraído mucha atención de los medios de comunicación), sino también sobre las personas de edad avanzada que quedaron atrás y sobre los migrantes que regresan a casa, dos figuras ausentes por lo general del debate público sobre la emigración. Se prestó especial atención a la elaboración de las preguntas, a fin de evitar un refuerzo del estigma que circula en los países de Europa del Este, que representa a la emigración (especialmente si es femenina) y a las familias transnacionales como una de las principales causas de la desintegración social (Vianello, 2011; Lutz \& Palenga-Möllenbeck, 2012).

\section{LOS MENORES Y LAS PERSONAS MAYORES QUE SE QUEDAN «ATRÁS»}

Las migraciones de mujeres y hombres adultos generan en los países de origen un incremento de los menores que se quedan sin uno o ambos padres y de los ancianos sin sus hijos/as.

El Ministerio de Educación y Juventud moldavo recoge anualmente a través del sistema escolar los datos relativos a los menores de familias migrantes. Se estima que alrededor del 30\% de los niños 
moldavos vive sin uno o ambos padres a causa de la emigración, es decir, casi 200 mil menores con menos de 14 años (Sarbu 2007). Al 1 de abril de 2010 el Ministerio de Educación y el Consejo para la Protección de los Derechos de los niños calculaban que, entre los menores en edad escolar (7-18 años), aproximadamente 84 mil viven sin la supervisión de los padres; de éstos el 66\% tenía sólo un progenitor emigrante, mientras el 34\% tenía la madre y el padre en el exterior (HelpAge International, UNICEF 2010).

En 2012, la autoridad rumana para la protección de los niños registraba 83.500 hijos menores de padres emigrantes: 24 mil tenían ambos padres en el exterior, 10 mil provenían de una familia monoparental donde el único progenitor había emigrado y poco menos de 49 mil habían permanecido con uno de los dos padres. La mayoría de estos niños han sido encomendados a parientes de hasta cuarto grado de consanguineidad, mientras que sólo el $4,2 \%$ ha sido recogido por los servicios sociales (Ministerul Muncii, Familiei şi Protecţiei Sociale Direcţia Protecţia Copilului 2012).

Por último, en lo referente a Ucrania, según los datos del Ministerio de la Familia, Juventud y Deporte hay alrededor de $200 \mathrm{mil}$ menores con uno o ambos padres viviendo en el exterior (IOM Italy 2010). Algunas investigaciones llevadas a cabo a nivel local en las regiones de Chernivtsi, Ternopil, L'viv e Ivano-Frankivsk, caracterizadas por una importante migración femenina hacia Europa meridional, concluyen que alrededor de una cuarta parte de los niños de esas regiones es educado por sólo un progenitor (Soleterre, Zaporuka 2012).

En lo referente a los ancianos que permanecen sin el sostén físico de uno o varios de sus hijos a causa de la emigración, no hay datos oficiales en ninguno de los tres países. Sin embargo, vistas las dimensiones del fenómeno de los menores que son encomendados a otros familiares, se puede afirmar que son también muchas las personas ancianas que se quedan solas. Además, un indicador de la amplitud del fenómeno puede ser el creciente interés por parte de la Organización Internacional para las Migraciones y de Help Age International por el problema de los así llamados «elderly left behind» (mayores que se quedan «atrás»), que se aborda conjuntamente con la preocupación por los niños «left behind» (HelpAge International, UNICEF 2010; Cheianu-Andrei et al. 2011). Las investigaciones realizadas recalcan que cuando ambos padres emigran los abuelos asumen el rol central en el cuidado de los menores, aunque para muchos ancianos 
es un compromiso - económico y humano- insostenible, porque a menudo carecen de los recursos necesarios o están enfermos. En los tres países estudiados los ancianos, en efecto, son una de las categorías sociales más vulnerables, a causa tanto de las bajas pensiones que reciben como por la frecuente negligencia en el cuidado de su salud, si bien están cubiertos formalmente por el sistema sanitario nacional (Lewis 2005; HelpAge International, UNICEF 2010).

\section{LAS FAMILIAS TRANSNACIONALES}

\subsection{La familia matryoshka y las mujeres de base}

En los tres países tomados en consideración, el cuidado de las personas dependientes es una tarea que concierne, en gran parte, a las familias. A continuación se detallan las prácticas que adoptan las familias transnacionales para sostener la reproducción cotidiana de los menores y de los ancianos que permanecen en los países de origen.

En la República de Moldavia, Rumanía y Ucrania las abuelas han desarrollado tradicionalmente un rol central como sostén de las hijas en las actividades domésticas y de cuidados (Pantea 2011). En particular, en los países ex soviéticos ha venido siendo común la así denominada «familia matryoshka», formada por tres generaciones de mujeres: abuela, hija y nieta (Poskanzer 1995). Este tipo de familia se basa en el intercambio de protección económica y cuidados entre las tres generaciones: en un primer momento la abuela se ocupa del trabajo de reproducción mientras la hija se dedica al trabajo retribuido, posteriormente, las tareas se invierten, la hija cuida de la madre anciana y la nieta se ocupa del sostén económico del grupo familiar.

En consecuencia, cuando en estos países la emigración pasa a ser una estrategia común de supervivencia, las familias se organizan siguiendo los mismos esquemas: las hijas de la generación del medio emigran y las abuelas permanecen en casa con los nietos y nietas. Sin embargo, los flujos migratorios ucranianos, rumanos y moldavos se caracterizan por estar formados por personas de diferentes franjas de edad, de modo que son diferentes también las edades de las abuelas y de los nietos que permanecen en el país de procedencia. 
De Rumanía y la República de Moldavia han emigrado numerosas mujeres jóvenes que han encomendado sus hijos a las abuelas aún relativamente jóvenes y capaces de cuidar de los nietos. Estas son las características de algunas de estas abuelas cuidadoras con las que se estableció contacto durante la realización del trabajo de campo. En Chişinau conocimos a Natalia, una mujer de 60 años que cuida a su nieto de 2 años mientras que su hijo y su nuera están en Italia. En Drochia reside María, una abuela de 78 años que, aunque ahora vive sola, cuidó a su nieta (la hija de su hija) desde los 63 hasta los 73 años. En Bucarest, nos recibieron dos familias en las que los nietos habían sido encomendados al cuidado de los abuelos y abuelas en los primeros años de su vida. En Iaşi hablamos con una abuela de 61 años a cargo de su nieta de 7 años.

Estos casos proporcionan información relevante sobre la selección de los abuelos y abuelas cuidadores. En primar lugar, enseñan que no hay una regla fija sobre qué abuelos son los que tienen que cuidar a los nietos cuando sus padres emigran, puesto que en ocasiones se trata de abuelos por parte de padre y en otras de abuelos por parte de madre. En segundo lugar, aunque en la mayoría de los casos son las abuelas las que llevan el principal papel de cuidadoras (Pantea 2011), los abuelos están presentes y cuidan a los nietos cuando las abuelas no pueden. Este aspecto se ve en particular en el relato del abuelo Petru, que estuvo solo con el niño durante 8 meses porque también su mujer había emigrado de Rumanía:

En el 2006 la madre se fue a España. [...] El niño tiene problemas de salud, si bien aparentemente parece normal. Nosotros hemos hecho todo lo que era necesario. En el 2008 mi mujer me dejó solo con el niño durante 8 meses porque se fue a Grecia a trabajar (Bucarest, septiembre de 2012).

También las emigrantes ucranianas encomiendan sus hijas e hijos a los abuelos o incluso a las tías, sin embargo el compromiso que se les requiere es diferente desde el punto de vista tanto temporal como cualitativo, porque, en general, las emigrantes son de mayor edad y tienen hijos más grandes que requieren un trabajo de cuidado diferente. Las tareas principales de las que se encargan estos abuelos son la gestión de la casa, la administración de las remesas de dinero, el control de la marcha escolar así como el cuidado 
afectivo de los nietos. Svitlana, por ejemplo, tiene 21 años y vive sola con su hermana menor. Su madre es divorciada y reside en Italia desde hace 6 años. Las dos hermanas han vivido durante algún tiempo con el padre y la abuela materna pero cuando alcanzaron la mayoría de edad ambos decidieron marcharse y dejarlas solas. La abuela que vive en el mismo barrio que las muchachas, no obstante, sigue administrando las remesas y controlando los gastos. Además, como se ha hecho mayor empieza a ser ella quien necesita ayuda de sus nietas. Se invierten las relaciones de cuidado y el rol de las abuelas se transforma de suministradores de asistencia en receptoras de cuidados. En el caso de Svitlana funciona un modelo matryoshka ligeramente modificado, dado que en ausencia de las hijas, es decir, de las mujeres de la generación del medio, el cuidado de las personas ancianas recae en las jóvenes nietas. Las hijas que están fuera, en efecto, intervienen sólo en casos excepcionales, mientras que en la vida cotidiana la relación de cuidado se instaura entre las abuelas y las nietas.

Mí madre sigue mandando dinero a la abuela, la abuela nos da el dinero y nosotras en un cuaderno escribimos en qué hemos gastado, qué hemos pagado y cuando mamá viene, se lo mostramos. Ahora el dinero para comer nos lo da papá, mamá en cambio manda dinero para pagar todos los recibos y los gastos de la escuela o la universidad. [...] Mi abuela tiene dos hijas, mi madre y mi tía que vive en Roma y no tiene hijos. Cuando la abuela nos necesita, vamos a ayudarla, vive aquí cerca. El año pasado la abuela se rompió una pierna y nosotras durante un tiempo nos mudamos a su casa pero después como yo iba a la universidad y mi hermana a la escuela no teníamos tiempo porque teníamos que estudiar. Así que mamá vino y estuvo durante unos meses hasta que le quitaron el yeso, primero vino mi tía y después mamá (L'viv, abril de 2012).

Las familias numerosas por lo general logran responder con mayor éxito a las nuevas necesidades de los menores y de los padres ancianos, porque hay más posibilidades de que por lo menos una hermana o un hermano, un hijo o una hija, haya permanecido en el país de origen. En estos casos los hermanos y hermanas que viven en el exterior pagan los gastos y los otros se ocupan de las cuestiones prácticas (Díaz Gorfinkiel \& Escrivá 2012). El relato de María es muy útil desde este punto de vista, ya que ella cuida tanto de sus 
nietas como de sus padres. Ella es «la mujer de base», es decir el pilar de la reorganización de los cuidados después de la emigración a Italia de su hermana y de su hermano.

Ambas hemos ejercido con ellas de madre, porque mi hermana y yo nos hemos ocupado juntas de las niñas. Han sido tres niñas con dos madres. [...] Me quedé con ellas 3 años [...] Si no hubiera tenido padres ancianos que cuidar, me hubiera ido yo igual al exterior, porque. mi hermano también se fue a Italia. La verdad es que yo soy la única que puede ocuparse de nuestros padres. Ha sido una decisión mía. Yo no he tenido hijos, mientras que ellos sí, por lo tanto, está bien así. Si hubiera tenido hijos hubiera sido distinto. [...] Yo soy la mujer de base (Galaţi, septiembre de 2012).

\subsection{Los cuidados a distancia}

La amplia y rica literatura sobre la maternidad transnacional demuestra que la mujeres que emigran siguen cuidando de los hijos e hijas que se quedaron "atrás», tanto materialmente, enviando dinero y regalos, como emocionalmente, a través de los viejos y nuevos medios de comunicación (cartas, correos electrónicos, mensajes telefónicos, llamadas gratis a través de Internet con Skype, etc.) (Kraler, et al, 2011; Carling et al, 2012).

Las remesas adquieren un profundo «significado simbólico» (Zelizer, 1997), puesto que para las migrantes son la manera de seguir presente en el país de origen (Sayad, 1999). Como muchos estudios han demostrado, el acto de envío de dinero es a menudo representado por las mujeres migrantes como un símbolo de amor y fidelidad hacia sus familias (Hondagneu-Sotelo, Avila 1997; Parreñas, 2005). Generalmente las remesas sirven para garantizar a los familiares una dieta equilibrada, un hogar cálido y confortable, atención médica especializada, y el acceso a la universidad. Además, una parte del dinero que han ganado en el extranjero a menudo se invierte en la renovación de las casas.

Las madres migrantes intentan asimismo seguir el rendimiento escolar de sus hijos e hijas, comunicándose con los profesores por teléfono o en persona. Sobre este tema es muy interesante el relato del director de una escuela bastante prestigiosa de Chişinau que 
corrobora la participación activa de las madres migrantes y enseña cómo se puede facilitar la comunicación entre familias migrantes y escuela:

Tenemos una base común de datos electrónicos con todos los registros de los estudiantes, que está colgada en nuestra página web. Los padres tienen un código para entrar y ver todos los datos. [...] Los padres que viven en el extranjero participan mucho. En mi escuela los padres tienen que venir a hablar con los profesores por lo menos una vez al año. Ahora estoy de vacaciones, pero yo vengo de todas formas de 9 a 10 para hablar con los padres. Hoy hablé con dos madres y un padre que viven en Italia y están aquí para las fiestas (Chişinau, agosto 2012).

La literatura acerca de las prácticas de cuidados a distancia de las personas mayores es mucho más limitada, en particular en lo referente a las migraciones del Este de Europa, mientras que existen investigaciones sobre otras área geográficas (King, Vullnetari 2006; Zechner 2006; Baldassar 2007; Baldassar et al. 2007; Mazzucato 2008). Cuando tanto hijas como nietas se encuentran todas en el exterior, los padres se sienten a menudo solos, esperando que alguno vuelva para cuidarlos. La señora Victoria, por ejemplo, tiene 62 años, es viuda y todos sus hijos residen en Italia. Durante siete años se ocupó de los nietos y ahora se quedó sola, viviendo en la casa de la hija. Por el momento es autónoma y su principal problema es la soledad, pero en perspectiva espera que alguno vuelva a casa cuando le haga falta.

Yo estoy contenta de que mis hijos en Italia estén bien y tengan trabajo, el problema es que me siento sola, siento su ausencia. Tengo dos hijos, una mujer y un varón, ambos están en Italia. Les veo una vez al año, en agosto, vuelven a casa, después hablamos por skype, por teléfono.[...] Siempre me mandan dinero, regalos, paquetes. Uva, kiwi, naranjas, mandarinas. Lo que pueden mandarme. Cuándo vienen acá, yo cocino para ellos. [ríe] Hablamos, visitamos monasterios.

Pensando en el futuro, cuando necesite ayuda, ¿de quién espera mayor ayuda?

De mi hija. [ríe] También mi hijo me ayudará. Pero como vivo en la casa de mi hija, espero más ayuda de su parte. Pienso que también ellos un día volverán a casa (Chişinau, agosto 2012). 
En estos casos se comprueba que los hijos cuidan de los padres a distancia. Les llaman por teléfono todos los días para asegurarse de que estén bien, les mandan el dinero necesario para satisfacer todas sus necesidades materiales y algunas veces pagan a personas, en general vecinos, para que les ayuden con los quehaceres de la vida cotidiana. Cuando los hijos vuelven durante sus vacaciones de verano, pasan bastante tiempo con los padres: arreglan la casa, les llevan al médico, les acompañan a visitar a los parientes, preparan las conservas para el invierno y así sucesivamente. Sin embargo, todavía no está claro qué prácticas adoptarán las mujeres y los hombres emigrantes cuando sus padres necesiten cuidados personales cotidianos. De las entrevistas realizadas sabemos que cuando un padre se enferma, las hijas vuelven al país de origen para cuidar de él y para organizar la asistencia que necesita. De hecho se observa la dificultad de que las hijas escojan reagrupar a los padres enfermos en el país de inmigración, ya sea por no causar otros traumas, ya por los complejos trámites burocráticos.

\section{EL ESTADO}

Si bien la familia es el principal proveedor de cuidados, también los otros pilares del sistema del diamante de los cuidados se están equipando para afrontar las nuevas necesidades sobrevenidas o evidenciadas aún más por las migraciones internacionales. Las políticas sociales dirigidas a los hijos de los emigrantes se han desarrollado, gracias al empeño de diferentes organizaciones internacionales (IOM y UNICEF) que durante años han denunciado el problema, corriendo el riesgo también de contribuir al proceso de estigmatización de las mujeres emigrantes (Vianello 2011; Lutz, Palenga-Möllenbeck 2012). En cambio, los servicios dedicados a los ancianos que se quedaron solos están poco avanzados, si bien en los últimos años se nota una atención creciente respecto a dicha categoría.

En Rumanía y en la República de Moldavia, el Estado desarrolla una función importante de recogida de datos a través del sistema escolar, que tiene como efecto indirecto también la sensibilización de los maestros respecto a los problemas de los hijos de los emigrantes. 
Además, como cuenta una maestra moldava, las escuelas tienen organismos que se encargan de la gestión de los casos difíciles.

Estamos realizando, en todas las escuelas, seguimiento de los hijos de los emigrantes para entender cómo ayudarles. Tenemos dos psicólogos. Somos un bachillerato grande con 1.200 estudiantes. Se realizan estudios a los niños y jóvenes que tienen sus padres en el exterior, a los que tienen problemas y a los normales. En todas las escuelas tenemos una comisión para los menores de edad, formada por un psicólogo, un policía, una vicedirectora y maestras. Nos reunimos una vez por semana para discutir sobre los problemas de los niños, de aquellos que no asisten a las clases, de aquellos con problemas (Chişinau, agosto 2012).

En las escuelas rumanas, en general, son las orientadoras quienes se ocupan mayormente de los problemas de los hijos de los emigrantes, porque tienen una tarea de soporte educativo y de facilitación de la comunicación entre los padres, maestros y estudiantes. En Ucrania, en cambio, aún no se ha puesto en marcha un sistema de medición del fenómeno de los hijos que se quedan «atrás». Sin embargo, también en este país, los maestros contactados durante la investigación se han distinguido por una particular sensibilidad, visto que gracias al contacto cotidiano con los niños son los primeros en darse cuenta de los problemas y de intervenir en su ayuda, incluso si no forma parte de sus tareas. La escuela es el lugar privilegiado donde se manifiestan las nuevas necesidades de atención a los niños que permanecen sin padres a causa de la emigración. En efecto, en Ucrania uno de los pocos programas nacionales sobre la cuestión específica de los hijos de los trabajadores emigrantes ha sido adoptado por el Ministerio de Educación y Ciencia (25/12/2007) para formar a psicólogos y educadores respecto a los problemas de esta categoría (IOM-Italia 2010).

En Rumanía y en la República de Moldavia se han aprobado leyes $^{3}$ que obligan a los padres que se marchan al extranjero a nombrar un tutor legal para sus hijos y encomiendan a los servicios sociales la tarea de controlar su custodia. En la República de Moldavia ha sido adoptado, además, el «Plan Nacional de Acción para la protección de los niños que no disponen de los cuidados de los padres

3 Se trata de la ley n. 180/10-07-2008 para la República de Moldavia y la ley n. 219/2006 para Rumanía. 
2010-2011» que incluye a los hijos de los emigrantes e identifica las líneas de intervención para reforzar las políticas dirigidas a esta categoría, en el plano legal, formativo, educativo, sanitario y de sensibilización de la opinión pública. Sin embargo, son numerosos los casos que escapan a los servicios sociales, ya sea porque los padres no comunican su partida y, por tanto, no designan un tutor, como porque el número de trabajadores sociales está subdimensionado respecto a las necesidades reales de intervención (Cheianu-Andrei et al. 2011).

Como se observa en la entrevista con un trabajador social, en Rumanía se ha elaborado un protocolo articulado para la protección de los hijos de los emigrantes que prevé que los servicios sociales se hagan cargo del menor y que se prepare un plan de intervención personalizado para cada niño.

En los casos donde la familia es monoparental, la madre o el padre está obligado a firmar antes de su partida un poder donde designa un tutor del niño y nosotros realizamos un seguimiento del niño. Cada dos o tres meses se realiza una supervisión, mientras el progenitor está trabajando en el exterior seguimos al niño en la escuela, también lo llevamos al médico, asesoramos a la familia, al niño y a la persona que se ocupa del niño. Como el poder tiene sólo un año de validez, en el caso de que la madre o el padre rompa el vínculo con el niño estamos obligados a tomar una decisión sobre el niño: o bien se establece una medida de protección o se lo ingresa en una institución de acogida (Iaşi, Septiembre 2012).

En lo referente a los ancianos, en cambio, no existen servicios dedicados a los padres de los emigrantes. En la República de Moldavia y en Ucrania, las políticas de bienestar dirigidas a los más pobres, en general, son limitadas, están sub-financiadas y dedicadas principalmente a servicios básicos (comedores y guarderías), por lo cual los padres de los emigrantes se ven a menudo excluidos, pues se entiende que gozan de las remesas. En la República de Moldavia, si bien existen programas de protección social para los ancianos en dificultad $^{4}$, la asistencia es insuficiente por la escasez de recursos

4 La estrategia para el desarrollo nacional 2008-2011 (ley n. 295 del 21-122007) y el Plan nacional para la creación de un sistema integrado de los servicios sociales (Decreto gubernamental n. 1512 del 31-12-2008). 
financieros y humanos. En particular, carecen de aquellos servicios que pueden sustituir la ausencia de los cuidados cotidianos de los familiares, es decir los servicios domiciliarios y los centros de día (Cheianu-Andrei et al. 2011). En cambio, en Rumanía las categorías de ancianos que tienen derecho a entrar en el sistema de asistencia social son más amplias, porque se incluye también a los ancianos que están solos a causa de la emigración de sus hijos ${ }^{5}$.

En síntesis, se observa una tendencia a la intervención estatal en casos extremos y no en forma preventiva como se hace para los menores, probablemente porque en Moldavia, Rumanía y Ucrania el valor social que se otorga a los niños como bien común es mayor que el que se otorga a las personas mayores.

\section{EL TERCER SECTOR}

Junto a las prestaciones netamente públicas destinadas a los menores que se quedan "atrás», en los países tomados en consideración, existen numerosas organizaciones del tercer sector que trabajan en este frente. Tales organizaciones ofrecen servicios sociales para los hijos de los emigrantes en general, en colaboración con las instituciones públicas. Esto ocurre especialmente en el caso rumano donde se ha previsto la estrecha colaboración entre los servicios sociales y las organizaciones del tercer sector para la puesta en marcha del plan de intervención personalizado (Luca, Gulei 2008). Entre las actividades más comunes se cuenta con: la organización de programas extra-escolares, la asistencia psicológica; la facilitación de la comunicación intra-familiar, el acompañamiento a los servicios socio-sanitarios, la ayuda económica. Además, en Rumanía se están desarrollando proyectos para apoyar a los menores que retornan al país de origen después de un período transcurrido en el exterior, que

5 En base a la ley 17/2000, las personas que tienen derecho a entrar en una de las siguientes categorías son quienes: están solos y no tienen familiares que se puedan ocupar de ellos; no tienen una vivienda y no disponen de los recursos económicos necesarios para garantizar su subsistencia; no gozan de una renta personal suficiente para hacerse cargo de sus cuidados; no son auto-suficientes y necesitan cuidados especializados; están imposibilitados para satisfacer sus necesidades a causa de una enfermedad (Luca, Gîrleanu- oitu 2012). 
prevén principalmente la asistencia psicológica y el apoyo para la reinserción escolar.

Ucrania se distingue, en particular, por haber desarrollado proyectos de cooperación descentralizada con Italia que se basan en la convicción de que es necesaria una política social transnacional que pueda dar respuestas adecuadas a las necesidades que se detectan en los dos polos del proceso migratorio (Piperno, Tognetti 2012). Un ejemplo emblemático de aplicación de dicho enfoque es el proyecto de cooperación entre la ONG italiana Soleterre (Milán) y la Fundación ucraniana Zaporuka (L'viv) destinado al sostén de las familias transnacionales. Ambas organizaciones ofrecen un servicio psicológico, social y jurídico, el primero dirigido principalmente a las mujeres emigrantes, el segundo a los familiares que permanecen en el país de origen (Soleterre, Zaporuka 2013).

En los países mencionados están también surgiendo nuevos servicios para los ancianos, aunque no específicos para los familiares de trabajadores migrantes, que son gestionados por organizaciones del tercer sector, tales como asilos, servicios domiciliarios, centros sociales, o asistencia psicológica, en los que se observa una significativa presencia de padres de emigrantes. Dichos servicios están, en efecto, cubriendo parcialmente la demanda de políticas sociales que, por el momento, está ampliamente insatisfecha en Ucrania y en la República de Moldavia, mientras que se satisface en parte en Rumanía a través de su integración en los servicios públicos existentes.

El único servicio para los ancianos que tienen hijos en el extranjero que hemos encontrado es el proyecto de la ONG rumana Alternative Sociale, que como cuenta un trabajador, ha nacido gracias a la experiencia laboral con los hijos de los emigrantes:

Hemos diseñado un proyecto partiendo precisamente de la práctica porque hemos visto en el campo niños que se quedaron solos, solos con los abuelos, y así nos hemos dado cuenta que no sólo los niños están solos sino también los padres mayores. [...] El problema principal que mencionan es que no tienen dinero. [...] Pero nosotros descubrimos que el dinero no es su único problema. No tienen amigos o parientes que les ayuden, tienen deudas porque no han pagado el alquiler o tienen problemas psicológicos o psiquiátricos. Se trata de ver, de entender quién puede ayudar a estas personas, cuáles son verdaderamente los problemas que tienen a partir de lo que nos cuentan (Iaşi, septiembre de 2012). 


\section{EL MERCADO}

También en los países de Europa oriental empiezan a emplearse cuidadoras informalmente (Hrženjak 2011), a pesar de que raramente las emplean las familias transnacionales. Para responder al déficit de cuidados está naciendo una oferta privada de servicios a las personas, aunque de manera limitada. Está penetrando lentamente en la cultura de las sociedades ucraniana, rumana y moldava, las figuras tan difundidas hoy por hoy en Italia y España de la asistente familiar a domicilio y de la niñera, a las cuales se les delega el cuidado de los ancianos y de los niños.

Durante la investigación se han identificado casos donde, en ausencia de otros familiares, los niños y los ancianos son puestos a cargo de cuidadores retribuidos mientras sus madres o hijas desarrollan un trabajo análogo en el exterior. Sin embargo, a diferencia de otras sociedades de emigración donde este sistema de reorganización de los cuidados está más difundido (véase por ejemplo el caso filipino estudiado por Parreñas 2000), en Europa oriental es un fenómeno aún limitado. Según los expertos consultados, su limitación es debida probablemente a la crisis económica, las menores diferencias salariales existentes entre los países de emigración e inmigración y a la escasa conveniencia en hacer este tipo de trabajo.

Antes de la crisis, las familias tenían más dinero, los que trabajaban en el extranjero enviaban más dinero, hacían mas visitas y pagaban a otras personas para que cuidasen de sus padres, bien un familiar o un vecino. Pero hoy en día no es así: las visitas disminuyeron, el dinero falta. Ahora no se lo pueden permitir, porque no trabajan o están endeudados. [...] Una mujer, una niñera a tiempo parcial gana 200 euros al mes, cuatro horas a la semana, así que muchas familias no se lo pueden permitir (Operadora de Alternative Sociale, Iaşi, septiembre de 2012).

Por último, en Rumanía se están abriendo hospitales privados para el ingreso a largo plazo de personas discapacitadas o muy enfermas y centros residenciales donde hay una significativa presencia de ancianos que se quedaron solos porque sus hijas y hijos emigraron, en cambio no se han hallado estructuras parecidas en Moldavia y Ucrania. Con todo, las familias migrantes rumanas que pueden 
permitirse esta categoría de servicios son muy pocas, como cuenta la directora de un hospital privado:

Por el momento, tengo sólo 10 personas de edad avanzada, y 7 de ellas tienen sus familias en el extranjero. Estas son las personas que pueden permitirse el lujo de quedarse aquí, pero cobro tarifas diferentes, en cierto modo se puede decir que decido el precio en función de las características de los pacientes y de las de sus familias, ya que si las hijas están trabajando en el extranjero como ayuda a domicilio yo se que no ganan mucho. Pero hay veces que aunque yo les haga descuento no se pueden permitir mis tarifas (Iaşi, septiembre de 2012).

En conclusión, analizando el desarrollo del mercado privado de los servicios de cuidados para niños y personas mayores se evidencian dos fenómenos. En primer lugar, una baja demanda de servicios debida a la poca disponibilidad económica de la mayoría de las familias transnacionales, generada sobre todo por la crisis económica. En segundo lugar, se evidencia la casi ausencia de servicios privados formales en Moldavia y Ucrania, probablemente a causa de la menor penetración de la economía de mercado en el sistema económico de las dos ex-Repúblicas soviéticas.

\section{CONCLUSIONES Y PERSPECTIVAS DE INVESTIGACIÓN}

La migración internacional desde Rumanía, Ucrania y la República de Moldavia hacia Europa Meridional está re-definiendo los regímenes de cuidados de los países de origen y de inmigración. En particular, el presente artículo se centra en lo que está sucediendo en los países de emigración. Hemos visto cómo los diferentes pilares del sistema del diamante de los cuidados se están reorganizando para responder a las nuevas necesidades sociales que surgen tras la emigración de amplias franjas de la población, en particular de la femenina. Las familias transnacionales son las primeras encargadas de poner en marcha nuevas prácticas para atender a los niños y a las personas mayores que permanecen en el país de origen, pero no están completamente solas. La re-definición de la organización de los 
cuidados está, en efecto, involucrando también otras instituciones, como el Estado, el tercer sector y el mercado.

En lo referente a las familias, hemos visto que los niños son encomendados principalmente a las abuelas, o como alternativa, a otros parientes de sexo femenino, siguiendo un sistema de organización del cuidado común en las sociedades de Europa oriental, caracterizado por la centralidad del rol de la abuela, aunque hay abuelos que cooperan activamente en las tareas de cuidado. Las madres emigrantes participan en el cuidado de los hijos a distancia, ocupándose predominantemente de su bienestar económico y emotivo. Sin embargo, para las abuelas, especialmente si son ancianas, la nueva carga de cuidado puede ser un compromiso excesivamente oneroso desde el punto de vista tanto humano como económico y puede producir efectos negativos en su estado de salud. Principalmente, en el caso ucraniano, que se caracteriza por una edad más avanzada tanto de los nietos como de las abuelas, es frecuente que en el transcurso de un breve período de tiempo la relación de cuidado se invierta, de modo que los nietos pasan a ocuparse de las abuelas en lugar de sus propias madres. Como alternativa, son las hermanas y más raramente los hermanos de los emigrantes quienes se ocupan del cuidado personal de los padres, mientras que quien está en el extranjero contribuye pagando gran parte de los gastos. Las situaciones más problemáticas se presentan cuando los miembros del grupo familiar no pueden cuidar de los ancianos porque viven todos en el extranjero o no disponen de los recursos para hacerlo. En estos casos, la persona anciana corre el riesgo de quedarse sola en condiciones de extrema vulnerabilidad.

Las prácticas adoptadas por las familias transnacionales no son siempre suficientes para cubrir la ausencia de la generación intermedia, por lo que a iniciativa de algunas organizaciones internacionales, se están desarrollando en los países de origen políticas sociales tendientes a reducir el impacto negativo de las migraciones. El objetivo al cual está dirigida gran parte de las prestaciones es a la atención de los menores que se quedan sin padres a causa de la emigración, pero en los últimos años están tomando forma también políticas para los ancianos con hijos e hijas en el exterior. Las instituciones públicas están renovando el estilo de sus servicios sociales y aumentando las colaboraciones con el tercer sector, que especialmente en algunas regiones de Rumanía es particularmente activo en la realización de proyectos para menores y ancianos en dificultad. 
Además, el caso ucraniano muestra otra transformación en curso, a saber, que también las políticas de bienestar están convirtiéndose en transnacionales, ya que aumenta la cooperación entre países de origen y destino en la realización de proyectos de sostén de las familias multi-locales. Por último, la emigración está favoreciendo de manera muy limitada el nacimiento del subsegmento de mercado de los servicios privados a las personas y, en particular, las prestaciones de cuidados.

En conclusión, se pueden identificar algunos indicios de cambio social respecto a los cuales sería deseable que se realizara una mayor investigación. En las familias transnacionales de Europa del este está en proceso una re-definición de las responsabilidades inter-generacionales de cuidados dictada por la falta de mujeres de la generación intermedia, que requeriría otras profundizaciones para comprender cómo se producen los procesos de negociación dentro de las familias según las relaciones de poder, cuáles son los ejes del conflicto y si y cómo están modificando las normas sociales compartidas relativas a los roles de género y generacionales (Tolstokorova 2010; Deneva 2012). Además, dentro de unos años, cuando los padres de los emigrantes sean más ancianos, será posible estudiar mejor las prácticas de cuidado de las familias transnacionales. Es posible, que una parte de las emigrantes escoja volver temporalmente al país natal y que otra parte decida llevar a los padres al país donde viven, como en el caso de las peruanas residentes en España estudiado por Díaz Gorfinkiel y Escrivá (2012). Por último, también serían necesarias otras investigaciones para comprender en qué medida la organización de los cuidados asociadas a los procesos migratorios están modificando los regímenes de cuidados ucraniano, rumano y moldavo en su globalidad.

\section{BIBLIOGRAFÍA}

Arango, J.; Bonifazi, C.; Finotelli, C.; Peixoto, J.; Sabino, C.; Strozza, S. y TRIANDAFYllidou, A. (2009): «The making of an immigration model: inflows, impacts and policies in Southern Europe». Idea Working Papers, $\mathrm{n}^{\circ} 9$.

Baldassar, L.; Baldock, C. y Wilding, R. (2007): Families Caring Across Borders: Migration, Aging and Transnational Caregiving. London, Palgrave MacMillan. 
Baldassar, L. y Merla, L. (2013): Transnational Families, Migration and the Circulation of Care: understanding mobility and absence in family life. London, Routledge.

Basch, L.; Glick Schiller, N. y Szanton Blanc, C. (1994): Nations unbound: Transnational projects, postcolonial predicaments and deterritorialized nation-states. New York, Gordon and Breach.

Bettio, F.; Simonazzi, A. y Villa, P. (2006): «Change in care regimes and female migration: the 'care drain' in the Mediterranean». Journal of European Social Policy, vol. 16, $\mathrm{n}^{\circ}$ 3, pp. 271-285.

Carling, J.; Menjívar, C. y Schmalzbauer, L. (2012): «Special Issue: Transnational Parenthood». Journal of Ethnic and Migration Studies, vol. 38, n 2 .

Cheianu-Andrei, D.; Gramma, R.; Milicenco, S.; Pritcan V.; Rusnac, V. y VacuLovschi, D. (2011): Specific needs of children and elderly left behind as a consequence of migration. Chisinau, CEP USM.

Deneva, N. (2012): «Transnational Aging Carers: On Transformation of Kinship and Citizenship in the Context of Migration among Bulgarian Muslims in Spain». Social Politics, vol. 19, n 1, pp. 105-128.

Diaz Gorfinkiel, M. y Escrivà, A. (2012): "Care of Older People in Migration Contexts: Local and Transnational Arrangements Between Peru and Spain». Social Politics, vol.19, n¹, pp. 129-141.

EHRENREICH B. y Hochschild A. R. (2003): Global women: nannies, maids and sex workers. New York Henry Hold \& Comapany.

Evers, J. (1996): "Part of the welfare mix: The third sector as an intermediate area between market, economy, state and community». Voluntas, vol. 6, n. 2, pp. 159-182.

FAIST, T. (2000): The volume and dynamics of international migration and transnational social spaces. Oxford, Oxford University Press.

Fodor, E.; Glass, C.; Kawachi, J. y Popescu, L. (2002): «Family policies and gender in Hungary, Poland, and Romania». Communist and Post-Communist Studies, vol. 35, pp. 475-490.

Guarnizo, L. E. у Sмiтh, M. P. (1998): Transnationalism from below. Piscataway Transaction Publishers.

HelpAge International, UNICEF (2010): Staying behind: the effects of migration on older people and children in Moldova. Chisinau.

Herrera, G. (2012): «Repensar el cuidado a través de la migraciòn internacional: mercado laboral, Estado y familias transnacionales en Ecuador». Cuadernos de Relaciones Laborales, vol. 20, n. 1, pp. 139-159.

Hondagneu-Sotelo, P. y Avila, E. (1997): «I'm Here, but I'm There: The Meanings of Latina Transnational Motherhood». Gender and Society, vol. 11, n. 5, pp. 548-571.

HRŽENJAK, M. (2011): «Revival of Informal Paid Care Work in Eastern European Countries». JäpPInen M., Kulmala M., SaARIne A., Gazing at Welfare, 
Gender and Agency in Post-Socialist Countries. Newcastle upon Tyne, Cambridge Scholars.

INE (2012): Estadística del Padrón Continuo a 1 de enero. Madrid, INE.

IOM Italy (2010): Final Report. Capacity building action towards Ukrainian local institution fo the empowerment of migratory and socio-educational policies on behalf of children, women and local communities. Disponible en http://www.childrenleftbehind.eu/wp-content/uploads/2011/11/FinalReport-ITA-UKR-project-IOM-Italy.pdf

IOM Moldova (2013): Children left behind issue in the limelight of the Moldovan and Italian authorities. Disponible en http://www.iom.md/index.php/en/media-center/press-releases/217-children-left-behind-issuein-the-limelight-of-the-moldovan-and-italian-authorities

Istat (2011): La popolazione straniera residente in Italia. Roma, Istat.

JENSON, J. (2003): Redesigning the "welfare mix» for families: policy challenges, Canadian Policy Research Networks, Ottawa.

King R. y Vullnetari J. (2006): «Orphan pensioners and migrating grandparents: the impact of mass migration on older people in rural Albania». Ageing \& Society, vol. 26, pp. 783-816.

Kofman, E. y Raghuram, P. (2009): «The Implications of Migration for Gender and Care Regimes in the South» en Social Policy and Development Programme, Paper Number 41.

Kraler, A.; Kofman, E.; Kohli, M. y Schmoll, C. (2011): Gender, Generations and the Family in International Migration, Amsterdam, Amsterdam University Press.

Laslett, B. y Brenner, J. (1989): «Gender and Social Reproduction: Historical Perspectives». Annual Review of Sociology, vol. 15, pp. 381-404.

Lewis A. Lipsitz (2005): «The Elderly People in Post-Soviet Ukraine: Medical, Social and Economic Challenges». Journal of The American Geriatrics Society, $\mathrm{n}^{\circ} 53$, pp. 2216-2220.

LuCA, C. y Girleanu- oitu, D. (2012): Working methodology for the psycho-social-juridical assistance of elders. Iași, Junumea Publishing House.

LuCA, C. y GuleI, A. (2008): Working methodology: social, psychological and juridical assistance for children left home alone as result of their parents going to work abroad. Iași, Terra Nostra.

Lutz, H. y Palenga-Möllenbeck, E. (2012): “Care Workers, Care Drain, and Care Chains: Reflections on Care, Migration, and Citizenship». Social Policy, vol. $19 \mathrm{n}^{\circ}$ 1, pp. 15-37.

Marchetti S. y Venturini, A. (2013): «Mothers and Grandmothers on the Move: Labour Mobility and the Household Strategies of Moldovan and Ukrainian Migrant Women in Italy». International Migration, Early View (Online Version of Record published before inclusion in an issue).

Mazzucato, V. (2008): "Transnational reciprocity: Ghanaian migrants and the care of their parents back home». En Alber E., Van der Geest S., 
Geissler W., Whyte S., Generations in Africa: Connections and Conflicts, pp. 111-133. Münster: LIT Verlag.

Ministerul MunciI, Familiei şi Protecţiei Sociale Direcţia Protecţia Copilului (2012): Situaţie copii cu părinţi plecaţi la muncă în străinătate 31.03.2012. Disponible en http://www.Copii.Ro/Alte_Categorii.Html

Orozco, A. (2010): Cadenas Globales de cuidado ¿Qué derechos para un régimen global de cuidados justo?. INSTRAW, NN.UU.

PANTEA, M. (2011): "Grandmothers as main caregivers in the context of parental migration». European Journal of Social Work, vol. 15, n 1, pp. 63-80.

PARreñas, R. (2000): «Migrant Filipina domestic workers and the international division of reproductive labor». Gender \& Society, vol. 14, n ${ }^{\circ}$, pp. 560-581.

Parreñas, R. S. (2005): Children of Global Migration. Transnational Families and Gendered Woes. Standford, Standford University Press.

Pascall, G. y Manning, N. (2000): «Gender and social policy: comparing welfare states in Central and Eastern Europe and the former Soviet Union». Journal of European Social Policy, vol. 10, n. 3, pp. 240-266.

Piperno, F. y Tognetti Bordogna, M. (2012): Welfare transnazionale. La frontiera esterna delle politiche sociali. Roma, Ediesse.

Poskanzer, A. (1995): «The matryoshka: the threegeneration soviet family in Israel». Coatemporary Family Therapy, vol. 17, n 4, pp. 413-427.

Raghuram, P. (2012): «Global care, local configurations - challenges to conceptualizations of care». Global Networks, vol. 12, n² 2, pp. 155-174.

Razavi, S. (2007): «The Political and Social Economy of Care in a Development Context». Gender and Development Program, paper $\mathrm{n}^{\circ} 3$.

SARBU A. (2007): Moldovan Children Struggle to Cope with their Parents' Economic Migration. New York, UNICEF.

SAYAD, A. (1999): La double absence. Des illusions de l'émigré aux souffrances de l'immigré. Pa-ris, Seuil.

SolEterre, Z. (2013): Reti migratorie femminili transnazionali tra Ucraina e Italia. Disponible en http://www.soleterre.org/it/info-center/pubblicazi one/19

Tache, I. y NeEsham, C. (2011): «The performance of welfare systems in PostCommunist Europe: the cases of Romania and Bulgaria». IJER, vol. 2, n. 5, pp. 90-107.

Tolstokorova, A. V. (2010): "Where Have All the Mothers Gone? The Gendered Effect of labour Migration and Transnationalism on the Institution of Parenthood in Ukraine», Anthropology of East Europe Review, vol. 28 , n. $1,184-214$. 
Vianello, F. A. (2011): “'Orfani sociali”: discorsi, rappresentazioni e politiche». En Sacchetto, D., Ai margini dell'Unione europea. Roma, Carocci, Roma, pp. 127-152.

Williams, F. (2010): «Migration and Care: Themes, Concepts and Challenges». Social Policy and Society, vol. 9, n 3, pp 385-396.

ZeChNER, M. (2008): «Care of older persons in transnational settings». Journal of Aging Studies, vol. 22, pp. 32-44.

Zelizer, V. (1997): The Social Meaning of Money. Princeton-Oxford, Princeton University Press. 